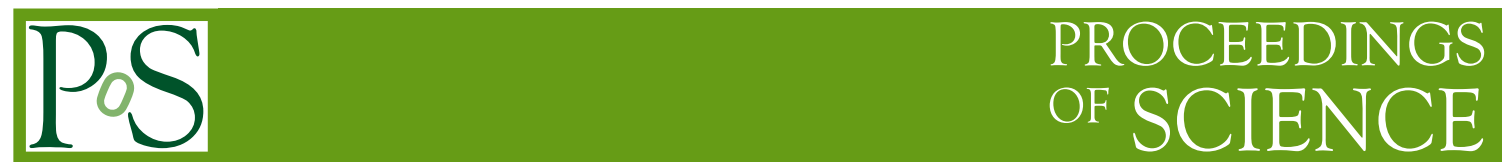

\title{
Rare decay searches at BABAR and Belle
}

\section{Fergus WILSON*}

STFC Rutherford Appleton Laboratory, Chilton, Didcot, Oxon, OX11 OQX, UK

E-mail: Fergus.Wilsonestfc.ac.uk

$B A B A R$ [1] and Belle [2] completed their data-taking in 2008 and 2010, respectively, but continue to publish results on rare $B$ decays. I report recent updates on searches for $B$ decays to invisible final states and the properties of the decay $B \rightarrow K^{(*)} \ell^{+} \ell^{-}$.

The XIth International Conference on Heavy Quarks and Leptons,

June 11-15, 2012

Prague, Czech Republic

* Speaker. 
1. $B^{0} \rightarrow$ invisible and $B^{0} \rightarrow$ invisible $+\gamma$

The rate for invisible $B$ decays is negligibly small within the Standard Model (SM), but can be larger in several models of new physics NP. The SM decay $B^{0} \rightarrow v \bar{v}$ is strongly helicity-suppressed by a factor of order $\left(m_{v} / m_{B^{0}}\right)^{2}$ [3] and the resulting branching fraction is well below the range of present experimental observability. The SM expectation for the $B^{0} \rightarrow v \bar{v} \gamma$ branching fraction is predicted to be of order $10^{-9}$, with very little uncertainty from hadronic interactions [4]. An experimental observation of an invisible $(+\gamma)$ decay of a $B^{0}$ with current experimental sensitivity would thus be a clear sign of physics beyond the SM.

The detection of invisible $B$ decays uses the fact that $B$ mesons are created in pairs, due to flavor conservation in $e^{+} e^{-}$interactions. If one $B$ is reconstructed in an event, one can thus infer that another $B$ has been produced. One approach is to reconstruct a $B^{0}$ decay to $D^{(*)}-\ell^{+} v$ (referred to as the "tag side"), then look for consistency with an invisible decay or a decay to a single photon of the other neutral $B$ (referred to as the "signal side"). The choice of reconstructing semileptonic $B^{0}$ decays on the tag side, with respect to fully-reconstructed $B^{0}$ hadronic final states, is motivated by a higher reconstruction efficiency. A disadvantage is the presence of the invisible neutrino, which prevents the exploitation of kinematic variables such as the reconstructed $B^{0}$ mass. However, the background contamination is offset by the presence of a high momentum lepton.

The $D^{*-}$ mesons are reconstructed in the final states $\bar{D}^{0} \pi^{-}$or $D^{-} \pi^{0}$, with $\bar{D}^{0}$ decays to $K^{+} \pi^{-}$, $K^{+} \pi^{-} \pi^{0}$, or $K^{+} \pi^{-} \pi^{+} \pi^{-}$, and $D^{-}$decays to $K^{+} \pi^{-} \pi^{-}$or $K_{S}^{0} \pi^{-}$. The $K_{S}^{0}$ mesons are reconstructed in the decay mode $K_{S}^{0} \rightarrow \pi^{+} \pi^{-}$, where the $\pi^{+} \pi^{-}$invariant mass lies in a $\pm 25 \mathrm{MeV} / c^{2}$ window around the nominal $K_{S}^{0}$ mass [5]. The $\pi^{0}$ candidates are composed of pairs of photons. Each photon must have a reconstructed energy above $30 \mathrm{MeV}$ in the laboratory frame, the sum of their energies must be greater than $200 \mathrm{MeV}$, and the $\pi^{0}$ candidates must have an invariant mass between 115 and $150 \mathrm{MeV} / c^{2}$. A mass-constrained fit is imposed on $\pi^{0}$ candidates in order to improve the resolution on the reconstructed invariant mass of the parent $D$ meson.

Kaon and pion candidates are then combined to reconstruct $D^{(*)}$ mesons. These are required to have an invariant mass within $60 \mathrm{MeV} / c^{2}$ of their nominal mass, except for $\bar{D}^{0}$ decays with a $\pi^{0}$ daughter, which must be within $100 \mathrm{MeV} / c^{2}$ of the nominal $\bar{D}^{0}$ mass. Mass-constrained fits are applied to $\bar{D}^{0}$ and $D^{-}$candidates in order to improve the measurement of the momentum of each $D$. The difference in reconstructed mass between $D^{*-}$ decay candidates and their $D$ daughters must be between $137 \mathrm{MeV} / c^{2}$ and $175 \mathrm{MeV} / c^{2}$. All $D^{(*)-}$ candidates must have a total momentum between 0.5 and $2.5 \mathrm{GeV} / c$ in the center-of-mass $\mathrm{CM}$ frame. Tracks selected as lepton candidates must pass either electron or muon selection criteria.

To further select $B^{0} \rightarrow D^{(*)-} \ell^{+} v$ candidates, a $D^{(*)-}$ candidate and a lepton candidate must be consistent with production at a common point in space. The decay vertex is reconstructed from a kinematic fit to all the candidate daughters, and a minimum $\chi^{2}$ vertex probability of 0.001 is required. The cosine of the angle between the $D^{(*)-} \ell^{+}$and the hypothesized $B^{0}$ candidate in the $\mathrm{CM}$ frame is calculated, under the assumption that the only particle missing is a neutrino:

$$
\cos \theta_{B, D^{(*)-\ell^{+}}}=\frac{2 E_{B} E_{D^{(*)-} \ell^{+}}-m_{B}^{2}-m_{D^{(*)-} \ell^{+}}^{2}}{2\left|\vec{p}_{B}\right|\left|\vec{p}_{D^{(*)-} \ell^{+}}\right|}
$$


The energy in the CM frame $E_{D^{(*)-} \ell^{+}}$and mass $m_{D^{(*)-} \ell^{+}}$of the $D^{(*)-} \ell^{+}$combination are determined from reconstructed momentum information, and $m_{B}$ is the nominal $B^{0}$ mass [5]. The $B^{0}$ momentum $\left|\vec{p}_{B}\right|$ and energy $E_{B}$ in the CM frame are determined from beam parameters. If the assumption that only a neutrino is missing in the $B^{0}$ decays is incorrect, then $\cos \theta_{B, D^{(*)-} \ell^{+}}$can fall outside the region $[-1,1]$. The $D^{(*)-} \ell^{+}$combination must satisfy $-5.5<\cos \theta_{B, D^{(*)-} \ell^{+}}<1.5$; this allows for non-physical $\cos \theta_{B, D^{(*)-} \ell^{+}}$values, accounting for detector energy and momentum resolution.

In order to reject background events where one charged or neutral particle is lost along the beam pipe, the cosine of the polar angle of the missing momentum in the $\mathrm{CM}$ frame is required to lie in the $[-0.9,0.9]$ range. The missing 4-momentum due to unreconstructed particles is defined as the difference between the $\Upsilon(4 S)$ and the reconstructed tag side 4-momentum. In the $B^{0} \rightarrow$ invisible $+\gamma$ channel, the signal-side photon 4-momentum is also subtracted from the $\Upsilon(4 S)$ one.

For the $B^{0} \rightarrow$ invisible decay, in events where the $D$ meson on the tag side decays into $K^{-} \pi^{+} \pi^{-}$, two additional selection criteria are applied. The first concerns the sum of the cosine of the angles between the kaon and two pions, $\cos \theta_{K \pi_{1}}+\cos \theta_{K \pi_{2}}>-0.8$, while the second concerns the sum of the cosine of the angles between the lepton and the pions, $\cos \theta_{\ell \pi_{1}}+\cos \theta_{\ell \pi_{2}}<0.8$. The main effect of this selection is the reduction of the background from mis-reconstructed $e^{+} e^{-} \rightarrow$ $\tau^{+} \tau^{-}$events.

To reconstruct $B^{0} \rightarrow$ invisible $+\gamma$ events, one remaining photon candidate with energy greater than $1.2 \mathrm{GeV}$ in the $\mathrm{CM}$ frame is also required. If the detected photon has an energy smaller than $1.2 \mathrm{GeV}$ in the $\mathrm{CM}$ frame, the event falls in the $B^{0} \rightarrow$ invisible category and the neutral candidate is considered as an extra photon in the event.

The signal region SR is defined as a $\pm 15 \mathrm{MeV} / c^{2}$ window around the nominal value for $m_{D}$ for the $B^{0} \rightarrow D^{-} \ell^{+} v$ sample, and as $0.139<\Delta m<0.148 \mathrm{GeV} / c^{2}$ for the $B^{0} \rightarrow D^{*-} \ell^{+} v$ sample. The excluded regions are used as the side band SB region.

A neural network $(\mathrm{NN})$ is used with up to six input observables to provide further discrimination between signal and background events [6]. The NN is trained using MC simulated signal samples, weighted off-peak data and MC simulated $B \bar{B}$ events. The selection on the output of the $\mathrm{NN}$ is optimized by minimizing the expected upper limit on the branching fraction, defined by a Bayesian approach, under the hypothesis of observing zero signal events.

The total energy in the calorimeter computed in the CM frame and not associated with neutral particles or charged tracks used in the $D^{(*)-} \ell^{+}$reconstruction is denoted as $E_{\text {extra. }}$. For $B^{0} \rightarrow$ invisible $+\gamma$, the energy of the highest-energy photon remaining in the event (the signal photon candidate) is also removed from the $E_{\text {extra }}$ computation. The $E_{\text {extra }}$ signal region is defined by imposing an upper bound at $1.2 \mathrm{GeV}$. In both $B^{0} \rightarrow$ invisible and $B^{0} \rightarrow$ invisible $+\gamma$ samples, this variable is strongly peaked near zero for signal, whereas for the background the distribution increases uniformly in the chosen signal region. Background events can populate the low $E_{\text {extra }}$ region, when charged or neutral particles from the event are either outside the fiducial volume of the detector or are unreconstructed due to detector inefficiencies. Contributions from mis-reconstructed $\pi^{0}$ decays usually populate the high $E_{\text {extra }}$ region.

Using detailed Monte Carlo simulations of $B^{0} \rightarrow$ invisible and $B^{0} \rightarrow$ invisible $+\gamma$ events, the signal efficiency is determined to be $(17.8 \pm 0.2) \times 10^{-4}$ for $B^{0} \rightarrow$ invisible and $(16.0 \pm 0.2) \times 10^{-4}$ for $B^{0} \rightarrow$ invisible $+\gamma$, where the uncertainties are statistical. 
The probability density functions (PDFs) are constructed for the $E_{\text {extra }}$ distribution for signal $\left(\mathscr{P}_{\text {sig }}\right)$ and background $\left(\mathscr{P}_{\text {bkg }}\right)$ using detailed MC simulation for signal and data from the $m_{D}$ and $\Delta m$ sidebands for background. The two PDFs are combined into an extended maximum likelihood function $\mathscr{L}$, defined as a function of the number of signal $N_{\text {sig }}$ and background events $N_{\text {bkg }}$.

The photon reconstruction has a detection lower energy bound of $30 \mathrm{MeV}$, and as a consequence, the $E_{\text {extra }}$ distribution is not continuous. To account for this effect, the likelihood is composed of two distinct parts, one for $E_{\text {extra }}>30 \mathrm{MeV}$ and one for $E_{\text {extra }}=0 \mathrm{MeV}$. The negative $\log$-likelihood is then minimized with respect to $N_{\mathrm{sig}}$ and $N_{\mathrm{bkg}}$ in the data sample. The fit returns $-22 \pm 9$ and $-3.1 \pm 5.2$ signal events for $B^{0} \rightarrow$ invisible and $B^{0} \rightarrow$ invisible $+\gamma$, respectively. Figure 1 shows the $E_{\text {extra }}$ distributions for $B^{0} \rightarrow$ invisible and $B^{0} \rightarrow$ invisible $+\gamma$ with the fit superimposed. The fitted signal yields are used to determine the decay branching fractions $\mathscr{B}$, which are defined as $\mathscr{B} \equiv N_{\text {sig }} /\left(\varepsilon \times N_{B \bar{B}}\right)$, where $\varepsilon$ is the total signal efficiency, corrected for data-MC discrepancies, and $N_{B \bar{B}}$ is the number of produced $B \bar{B}$ pairs. A Bayesian approach is used to set $90 \%$ confidence level (CL) upper limits on the branching fractions for $B^{0} \rightarrow$ invisible and $B^{0} \rightarrow$ invisible $+\gamma$. The resulting 90\% upper limits on the branching fractions are $\mathscr{B}\left(B^{0} \rightarrow\right.$ invisible $)<2.4 \times 10^{-5}$ and $\mathscr{B}\left(B^{0} \rightarrow\right.$ invisible $\left.+\gamma\right)<1.7 \times 10^{-5}[7]$.
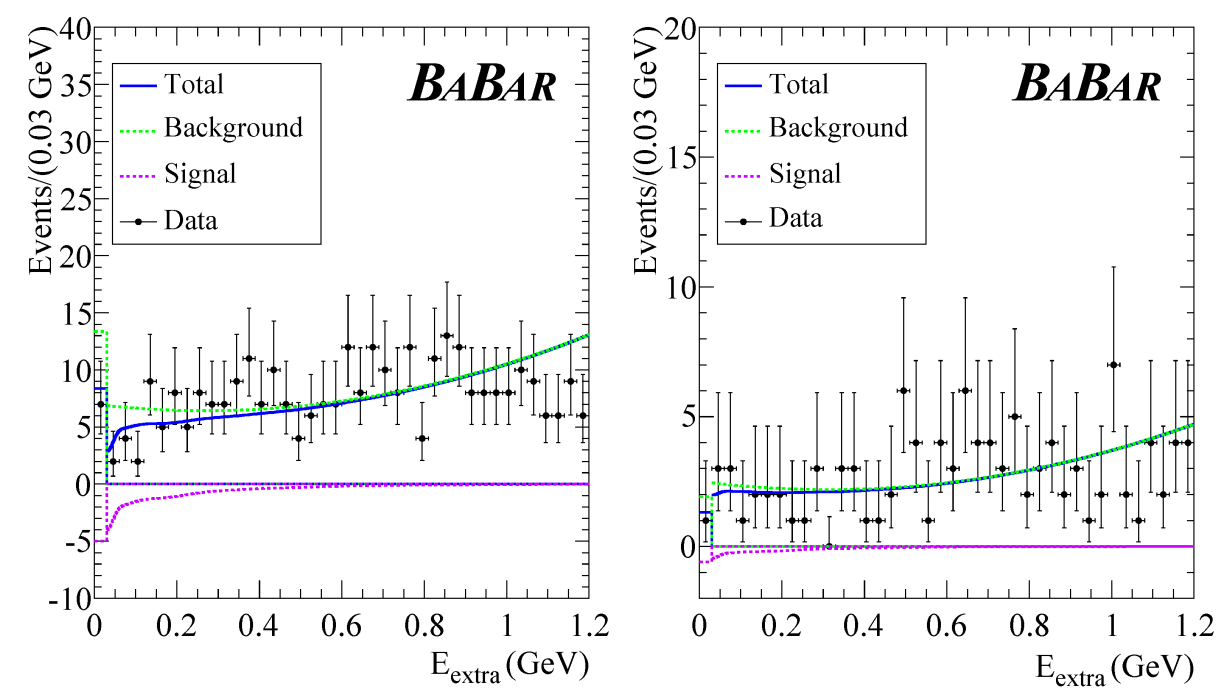

Figure 1: Results of the ML fit of $E_{\text {extra }}$ for $B^{0} \rightarrow$ invisible (left) and $B^{0} \rightarrow$ invisible $+\gamma$ (right) [7].

\section{2. $B \rightarrow K^{(*)} \ell^{+} \ell^{-}$}

The decays $B \rightarrow K^{(*)} \ell^{+} \ell^{-}$arise from flavor-changing neutral-current processes that are forbidden at tree level in the SM. The lowest-order SM processes contributing to these decays are the $\gamma / Z$ penguin loops and the $W^{+} W^{-}$box diagrams shown in Fig. 2. Their amplitudes are expressed in terms of hadronic form factors and perturbatively-calculable effective Wilson coefficients, $C_{7}^{\text {eff }}$, $C_{9}^{\text {eff }}$ and $C_{10}^{\text {eff }}$, which represent the electromagnetic penguin diagram, and the vector part and the axial-vector part of the linear combination of the $Z$ penguin and $W^{+} W^{-}$box diagrams, respec- 
tively [8]. In next-to-next-to-leading order (NNLO) at a renormalization scale $\mu=4.8 \mathrm{GeV}$, the effective Wilson coefficients are $C_{7}^{\text {eff }}=-0.304, C_{9}^{\text {eff }}=4.211$, and $C_{10}^{\text {eff }}=-4.103$ [9].
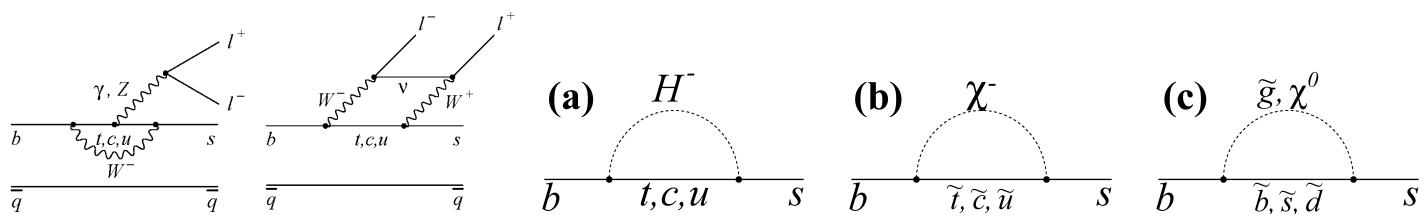

Figure 2: Left: lowest-order Feynman diagrams for $b \rightarrow s \ell^{+} \ell^{-}$; Right: examples of new physics loop contributions to $b \rightarrow s \ell^{+} \ell^{-}$: (a) charged Higgs $\left(H^{-}\right)$; (b) squark $(\tilde{t}, \tilde{c}, \tilde{u})$ and chargino $\left(\chi^{-}\right)$; (c) squark $(\tilde{b}, \tilde{s}, \tilde{d})$ and gluino $(\tilde{g}) /$ neutralino $\left(\chi^{0}\right)$.

New physics NP may add new penguin and box diagrams, which can contribute at the same order as the SM diagrams, as shown in Fig. 2. These contributions can modify the Wilson coefficients from their SM expectations. New contributions from scalar, pseudoscalar, and tensor currents may arise that can modify, in particular, the lepton-flavor ratios.

The $B \rightarrow K \ell^{+} \ell^{-}$and $B \rightarrow K^{*} \ell^{+} \ell^{-}$total branching fractions are predicted to be $(0.35 \pm 0.12) \times$ $10^{-6}$ and $(1.19 \pm 0.39) \times 10^{-6}$ (for $s>0.1 \mathrm{GeV}^{2} / c^{4}$, where $s$ in the invariant mass squared of the dilepton system, $m_{\ell^{+} \ell^{-}}^{2}$ ), respectively [10]. The $\sim 30 \%$ uncertainties are due to a lack of knowledge about the form factors that model the hadronic effects in the $B \rightarrow K^{(*)}$ transitions. Thus, measurements of decay rates to exclusive final states are less suited to searches for NP than rate asymmetries, where many theory uncertainties cancel.

The direct $C P$ asymmetry $\mathscr{A}_{C P}^{K^{(*)}}$ for charged and neutral $B$ decays flavor-tagged through $K^{*} \rightarrow$ $K^{+} \pi^{-}$and the ratio of rates to dimuon and dielectron final states $\mathscr{R}_{K^{(*)}}$ are defined as

$$
\mathscr{A}_{C P}^{K^{(*)}} \equiv \frac{\mathscr{B}\left(\bar{B} \rightarrow \bar{K}^{(*)} \ell^{+} \ell^{-}\right)-\mathscr{B}\left(B \rightarrow K^{(*)} \ell^{+} \ell^{-}\right)}{\mathscr{B}\left(\bar{B} \rightarrow \bar{K}^{(*)} \ell^{+} \ell^{-}\right)+\mathscr{B}\left(B \rightarrow K^{(*)} \ell^{+} \ell^{-}\right)}, \quad \mathscr{R}_{K^{(*)}} \equiv \frac{\mathscr{B}\left(B \rightarrow K^{(*)} \mu^{+} \mu^{-}\right)}{\mathscr{B}\left(B \rightarrow K^{(*)} e^{+} e^{-}\right)} .
$$

In the SM, $\mathscr{A}_{C P}^{K^{(*)}}$ is expected to be $\mathscr{O}\left(10^{-3}\right)$ while $\mathscr{R}_{K^{(*)}}$ is expected to be unity to within a few percent [12] for dilepton invariant masses above the dimuon kinematic threshold. However, $\mathscr{A}_{C P}^{K^{(*)}}$ may receive a significant enhancement from NP contributions at the electro-weak scale [11]. In two-Higgs-doublet models, including supersymmetry, $\mathscr{R}_{K^{(*)}}$ is sensitive to the presence of a neutral Higgs boson. When the ratio of the neutral Higgs field vacuum expectation values $\tan \beta$ is large, $\mathscr{R}_{K^{(*)}}$ might be increased by up to $10 \%$ [13].

The $C P$-averaged isospin asymmetry $\mathscr{A}_{I}^{K^{(*)}}$ is defined as

$$
\mathscr{A}_{I}^{K^{(*)}} \equiv \frac{\mathscr{B}\left(B^{0} \rightarrow K^{(*) 0} \ell^{+} \ell^{-}\right)-r_{\tau} \mathscr{B}\left(B^{+} \rightarrow K^{(*)+} \ell^{+} \ell^{-}\right)}{\mathscr{B}\left(B^{0} \rightarrow K^{(*) 0} \ell^{+} \ell^{-}\right)+r_{\tau} \mathscr{B}\left(B^{+} \rightarrow K^{(*)+} \ell^{+} \ell^{-}\right)}
$$

where $r_{\tau} \equiv \tau_{B^{0}} / \tau_{B^{+}}=1 /(1.071 \pm 0.009)$ is the ratio of $B^{0}$ and $B^{+}$lifetimes [5]. $\mathscr{A}_{I}^{K^{*}}$ has a SM expectation of $+6 \%$ to $+13 \%$ as $s \rightarrow 0$ [14]. This is consistent with the measured asymmetry $3 \pm 3 \%$ in $B \rightarrow K^{*} \gamma$ [15]. A calculation of the predicted $K^{*+}$ and $K^{* 0}$ rates integrated over the low $s$ region yields $\mathscr{A}_{I}^{K^{*}}=-0.005 \pm 0.020[16,17]$. In the high $s$ region, contributions from charmonium states are expected as an additional source of isospin asymmetry. However the measured asymmetries in the $J / \psi K^{(*)}$ and $\psi(2 S) K^{(*)}$ modes are all below $5 \%$ [5]. 
The longitudinal polarization $F_{L}$ and Forward-Backward asymmetry $A_{F B}$ are defined as

$$
\begin{aligned}
\frac{1}{\Gamma(s)} \frac{d}{d \cos \theta_{K}} & =\frac{3}{2} F_{L}(s) \cos ^{2} \theta_{K}+\frac{3}{4}\left(1-F_{L}(s)\right)\left(1-\cos ^{2} \theta_{K}\right) \\
\frac{1}{\Gamma(s)} \frac{d}{d \cos \theta_{l}} & =\frac{3}{4} F_{L}(s)\left(1-\cos ^{2} \theta_{l}\right)+\frac{3}{8}\left(1-F_{L}(s)\right)\left(1+\cos ^{2} \theta_{l}\right)+A_{F B} \cos \theta_{l}
\end{aligned}
$$

where $\theta_{K}$ is the angle of the $K$ momentum relative to the $B$ momentum in the $K^{*}$ rest frame and $\theta_{l}$ is the angle between the $\ell^{+}\left(\ell^{-}\right)$and the $B(\bar{B})$ momentum in the dilepton rest frame.

$B \rightarrow K^{(*)} \ell^{+} \ell^{-}$signal events are reconstructed in eight final states where $\ell=\mu$ or $e$, and $K^{(*)}$ is a $K_{S}^{0}, K^{+}, K^{* 0}$, or $K^{*+}$. The $K_{S}^{0}$ candidates are reconstructed in the $\pi^{+} \pi^{-}$final state. The $K^{(*)} h^{ \pm} \mu^{\mp}$ final states, where $h$ is a charged track with no particle identification requirement applied, are used to characterize backgrounds from hadrons misidentified as muons. The $K^{*} e^{ \pm} \mu^{\mp}$ sample is used to model the random combinatorial $m_{K \pi}$ background distributions. In each mode, the kinematic variables $m_{\mathrm{ES}}=\sqrt{E_{\mathrm{CM}}^{2} / 4-p_{B}^{* 2}}$ and $\Delta E=E_{B}^{*}-E_{\mathrm{CM}} / 2$ are utilized, where $p_{B}^{*}$ and $E_{B}^{*}$ are the $B$ momentum and energy in the $\Upsilon(4 S) \mathrm{CM}$ frame, and $E_{\mathrm{CM}}$ is the total $\mathrm{CM}$ energy. A fit region $m_{\mathrm{ES}}>5.2 \mathrm{GeV} / c^{2}$ is defined. The mass of the $K^{*}$, if present, must be in the region $0.72<m_{K \pi}<$ $1.10 \mathrm{GeV} / c^{2}$. The sideband $5.20<m_{\mathrm{ES}}<5.27 \mathrm{GeV} / c^{2}$ is used to characterize combinatorial background shapes and normalizations. For both the $e^{+} e^{-}$and $\mu^{+} \mu^{-}$modes, the $J / \psi(2.85<$ $\left.m_{\ell \ell}<3.18 \mathrm{GeV} / c^{2}\right)$ and $\psi(2 S)\left(3.59<m_{\ell \ell}<3.77 \mathrm{GeV} / c^{2}\right)$ mass regions are rejected. The vetoed events provide high-statistics control samples that are used to validate the fit methodology.

The main backgrounds arise from random combinations of leptons from semileptonic $B$ and $D$ decays. These combinatorial backgrounds from either $B \bar{B}$ events (referred to as " $B \bar{B}$ backgrounds") or continuum $q \bar{q}$ events ( $e^{+} e^{-} \rightarrow q \bar{q}, q=u, d, s, c$, referred to as " $q \bar{q}$ backgrounds") are suppressed using bagged decision trees (BDTs) [18]. A total of eight BDTs are trained in regions separated by $e^{+} e^{-} / \mu^{+} \mu^{-}$mode, low/high $s$, and $B \bar{B} / q \bar{q}$ backgrounds.

A significant source of background for $\mu^{+} \mu^{-}$final states arises from $B \rightarrow D\left(\rightarrow K^{(*)} \pi\right) \pi$ decays if both pions are misidentified as muons. The misidentification rates for muons is much higher than for electrons ( $\sim 3 \%$ and $\lesssim 0.1 \%$ per lepton, respectively). For these events, the invariant mass of the $K^{(*)} \pi$ system must be outside the range $1.84-1.90 \mathrm{GeV} / c^{2}$ after assigning the pion mass hypothesis to the muon candidates. Any remaining residual backgrounds from this type of contribution are parameterized using control samples obtained from data.

After applying all selection criteria about $85 \%$ of signal events contain more than one $B$ candidate. These candidates differ typically in one charged or neutral hadron. The average number of candidates per signal event is about six. To choose the best candidate, the following ratio is defined

$$
\mathscr{R} \equiv \frac{\mathscr{P}_{s i g}^{B \bar{B}}+\mathscr{P}_{s i g}^{q \bar{q}}}{\mathscr{P}_{s i g}^{B \bar{B}}+\mathscr{P}_{s i g}^{q \bar{q}}+\mathscr{P}_{b k g}^{B \bar{B}}+\mathscr{P}_{b k g}^{q \bar{q}}},
$$

where $\mathscr{P}_{\text {sig }}$ and $\mathscr{P}_{b k g}$ are probabilities calculated from the corresponding $B \bar{B}$ and $q \bar{q}$ BDT output distributions for signal and background, respectively. The candidate with the largest $\mathscr{R}$ is selected. The probability for a correctly-reconstructed signal event to be selected as the best candidate is mode-dependent and varies between about $80 \%$ and $95 \%$ for $s$ bins below the $J / \psi$ mass, while for $s$ bins above the $\psi(2 S)$ mass it varies between about $60 \%$ and $90 \%$. 
One-dimensional maximum likelihood (ML) fits are performed in $m_{\mathrm{ES}}$ for $K \ell^{+} \ell^{-}$modes and two-dimensional fits in $m_{\mathrm{ES}}$ and $m_{K \pi}$ for $K^{*} \ell^{+} \ell^{-}$modes to extract the signal yields. Appropriate PDFs are chosen to model the signal (using data from the vetoed $J / \psi$ events); combinatoric background; $B \rightarrow D\left(\rightarrow K^{(*)} \pi\right) \pi$ decays where both pions are misidentified as muons; signal crossfeed events from mis-reconstructed signal events; exclusive $B$ hadronic decays mis-reconstructed as $B \rightarrow K^{(*)} \ell^{+} \ell^{-}$; charmonium events that pass the veto; and peaking hadronic $B \rightarrow K^{*} \pi^{0}$ and $B \rightarrow K^{*} \eta$ decays. A simultaneous fits across different $K^{(*)} \ell^{+} \ell^{-}$modes in each $s$ bin is performed. Since efficiency-corrected signal yields are shared across various decay modes, rate asymmetries can be extracted directly from the fits. The fitted signal yields in $B^{+}$modes are corrected by the lifetime ratio $\tau_{B^{0}} / \tau_{B^{+}}$. The fit methodology is validated with charmonium control samples obtained from the dilepton mass regions around the $J / \psi$ and $\psi(2 S)$ resonances that are vetoed in the $B \rightarrow K^{(*)} \ell^{+} \ell^{-}$analysis. The fitting procedure is also validated by applying it to charmonium events to extract the rate asymmetries and angular observables. The measured $C P$ asymmetries $\mathscr{A}_{C P}$, lepton-flavor ratios $\mathscr{R}_{K^{(*)}}$ and isospin asymmetries $\mathscr{A}_{I}$ are in good agreement with SM expectations or world averages for $\mathscr{A}_{I}$.

Figure 3 shows the BABAR [19] and Belle [20] results for the partial branching fractions of the $K \ell^{+} \ell^{-}$and $K^{*} \ell^{+} \ell^{-}$modes in comparison to the results from the CDF Collaborations [21] and to the prediction of the Ali et. al. model [10]. The results are seen to agree with each other and also with recent partial branching fraction measurements of $B^{0} \rightarrow K^{* 0} \mu^{+} \mu^{-}$from LHCb [22].
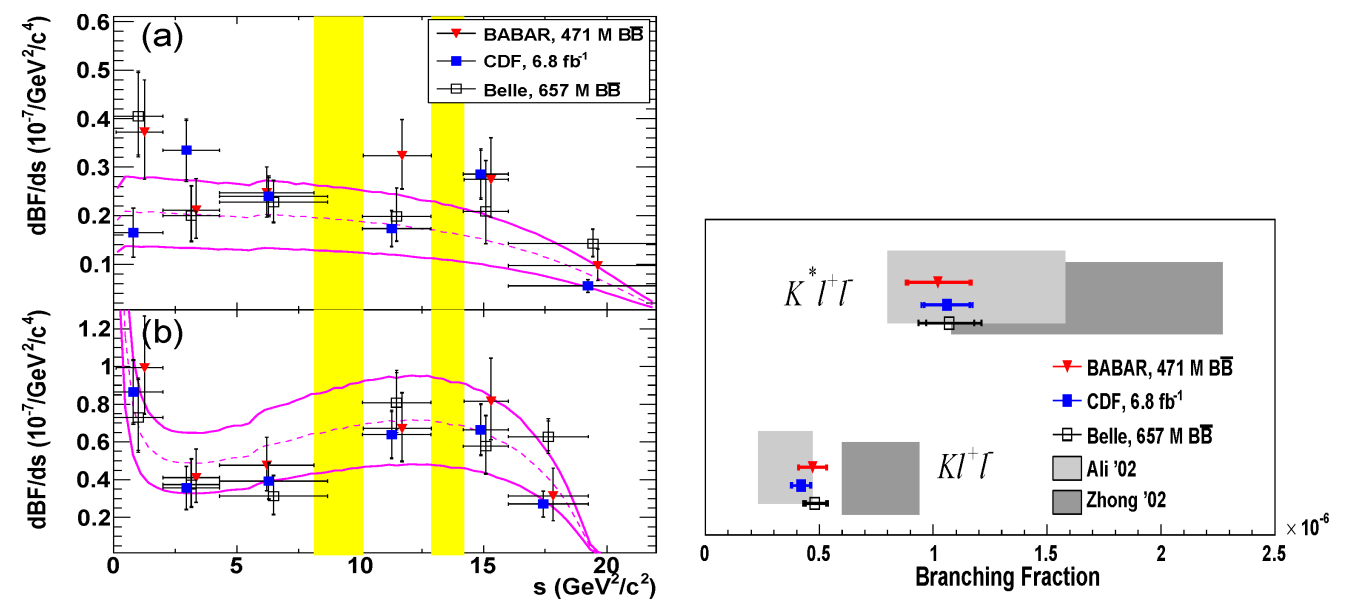

Figure 3: Left: partial branching fractions for the (a) $K \ell^{+} \ell^{-}$and (b) $K^{*} \ell^{+} \ell^{-}$modes as a function of $s$ showing BABAR measurements [19] (red triangles), Belle [20] (open squares), CDF [21] (blue solid squares), and the SM prediction from Ali et. al. [10] with $B \rightarrow K^{(*)}$ form factors [24] (magenta dashed lines). The magenta solid lines show the theory uncertainties. The vertical yellow shaded bands show the vetoed $s$ regions around the $J / \psi$ and $\psi(2 S)$. Right: total branching fractions for the $K \ell^{+} \ell^{-}$and $K^{*} \ell^{+} \ell^{-}$modes compared with predictions from Ali et. al. [10] (light grey bands), and Zhong et. al. [23] (dark grey bands) models.

$B A B A R$ measures the total branching fractions to be $\mathscr{B}\left(B \rightarrow K \ell^{+} \ell^{-}\right)=(4.7 \pm 0.6 \pm 0.2) \times 10^{-7}$ and $\mathscr{B}\left(B \rightarrow K^{*} \ell^{+} \ell^{-}\right)=\left(10.2_{-1.3}^{+1.4} \pm 0.5\right) \times 10^{-7}$. Here, the first uncertainties are statistical, and the second are systematic. The total branching fractions are shown in Fig. 3 in comparison to 
measurements from Belle [20] and CDF [21] and predictions from Ali et. al. [10] and Zhong et. al. [23].

To measure direct $\mathscr{A}_{C P}$, the $B$ and $\bar{B}$ samples are fitted in the two $K^{+} \ell^{+} \ell^{-}$modes and the four $K^{*} \ell^{+} \ell^{-}$modes. The measurements are performed in the full $s$ region, as well as in the low $s$ and high $s$ regions separately. The results are consistent with the SM expectation of negligible direct $\mathscr{A}_{C P}$.

The $e^{+} e^{-}$and $\mu^{+} \mu^{-}$samples are fitted in the four $K \ell^{+} \ell^{-}$modes and four $K^{*} \ell^{+} \ell^{-}$modes in the low $s$ and high $s$ regions separately to measure the lepton-flavor ratios. Figure 4 show $\mathscr{R}_{K}$ and $\mathscr{R}_{K^{*}}$ for $s>0.1 \mathrm{GeV}^{2} / c^{4}$. The BABAR results are consistent with unity as expected in the SM.
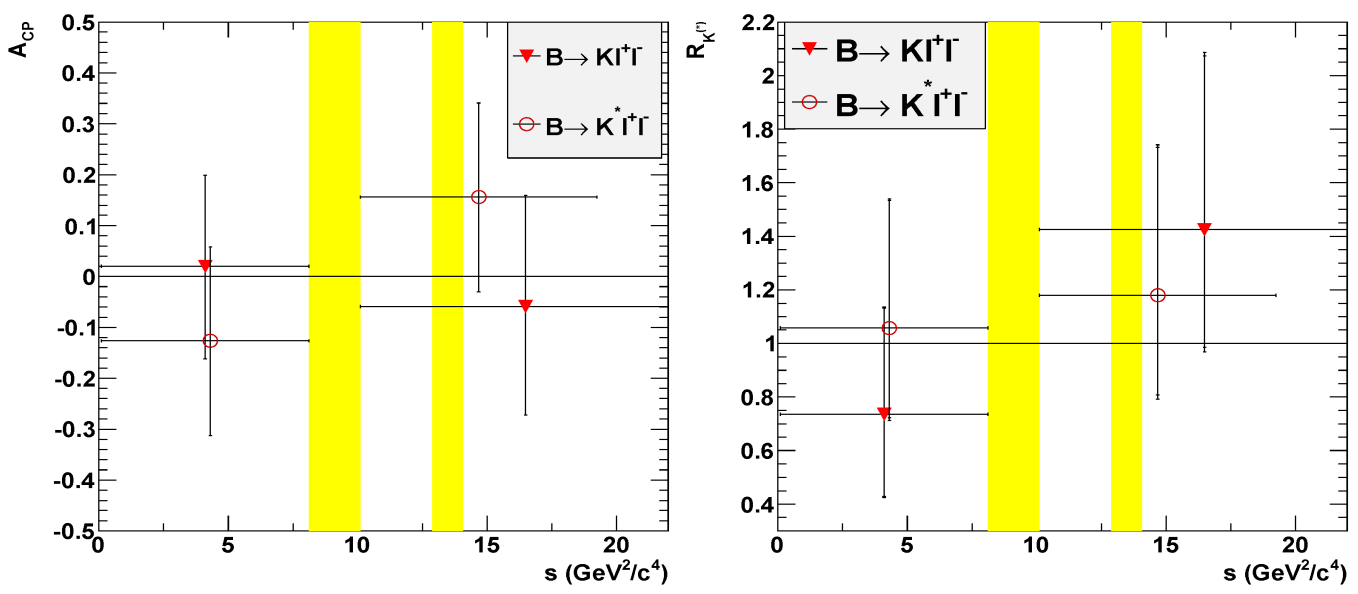

Figure 4: $C P$ asymmetries $\mathscr{A}_{C P}$ (left) and Lepton flavor ratios $\mathscr{R}_{K^{(*)}}$ (right) from $B A B A R$ [19] for $K \ell^{+} \ell^{-}$ modes (red solid triangles) and $K^{*} \ell^{+} \ell^{-}$modes (red open circles) as a function of $s$. The vertical yellow shaded bands show the vetoed $s$ regions around the $J / \psi$ and $\psi(2 S)$.

The data in each $s$ bin is fitted separately to determine $\mathscr{A}_{I}$ for the four combined $K \ell^{+} \ell^{-}$and the four combined $K^{*} \ell^{+} \ell^{-}$modes. Figure 5 shows the BABAR and Belle [20] measurements as a function of $s$. The two sets of results are seen to agree within the uncertainties. The BABAR results [19] are also consistent with the SM prediction that $\mathscr{A}_{I}$ is slightly negative $(\sim-1 \%)$ except in bin $s_{1}$, where it is predicted to have a value around $+5 \%$ [14].

The BABAR $\mathscr{A}_{I}$ measurements in the low $s$ region $\left(0.10<s<8.12 \mathrm{GeV}^{2} / c^{4}\right)$ yield $\mathscr{A}_{I}^{\text {low }}(B \rightarrow$ $\left.K \ell^{+} \ell^{-}\right)=-0.58_{-0.37}^{+0.29} \pm 0.02[2.1 \sigma]$ and $\mathscr{A}_{I}^{\text {low }}\left(B \rightarrow K^{*} \ell^{+} \ell^{-}\right)=-0.25_{-0.17}^{+0.20} \pm 0.03[1.2 \sigma]$. The $\mathscr{A}_{I}$ significances shown in the square brackets include all systematic uncertainties. The significance is estimated by refitting the data with $\mathscr{A}_{I}$ fixed to zero and computing the change in the log likelihood $\sqrt{2 \Delta \ln \mathscr{L}}$ between the nominal fit and the null hypothesis fit.

Figure 6 shows the longitudinal polarization $F_{L}$ and Forward-Backward asymmetry $A_{F B}$ results as a function of $s$. A common value of $F_{L}$ is first extracted from a fit to the data (see Eq. 2.3); this value is then used to extract $A_{F B}$ In both cases taking, the reconstruction efficiency as a function of $s$ and $\theta$ is taken into account. For $F_{L}$, there is a tendency for all the measurements to fall below the theory prediction. 


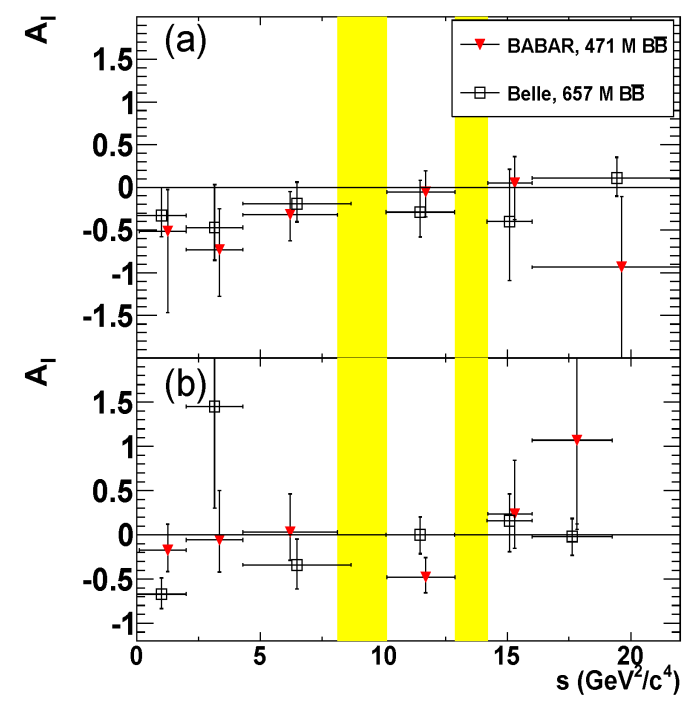

Figure 5: Isospin asymmetry $\mathscr{A}_{I}$ for the (a) $K \ell^{+} \ell^{-}$and (b) $K^{*} \ell^{+} \ell^{-}$modes as a function of $s$ for BABAR [19] (red triangles) and Belle [20] (open squares). The vertical yellow shaded bands show the vetoed $s$ regions around the $J / \psi$ and $\psi(2 S)$.
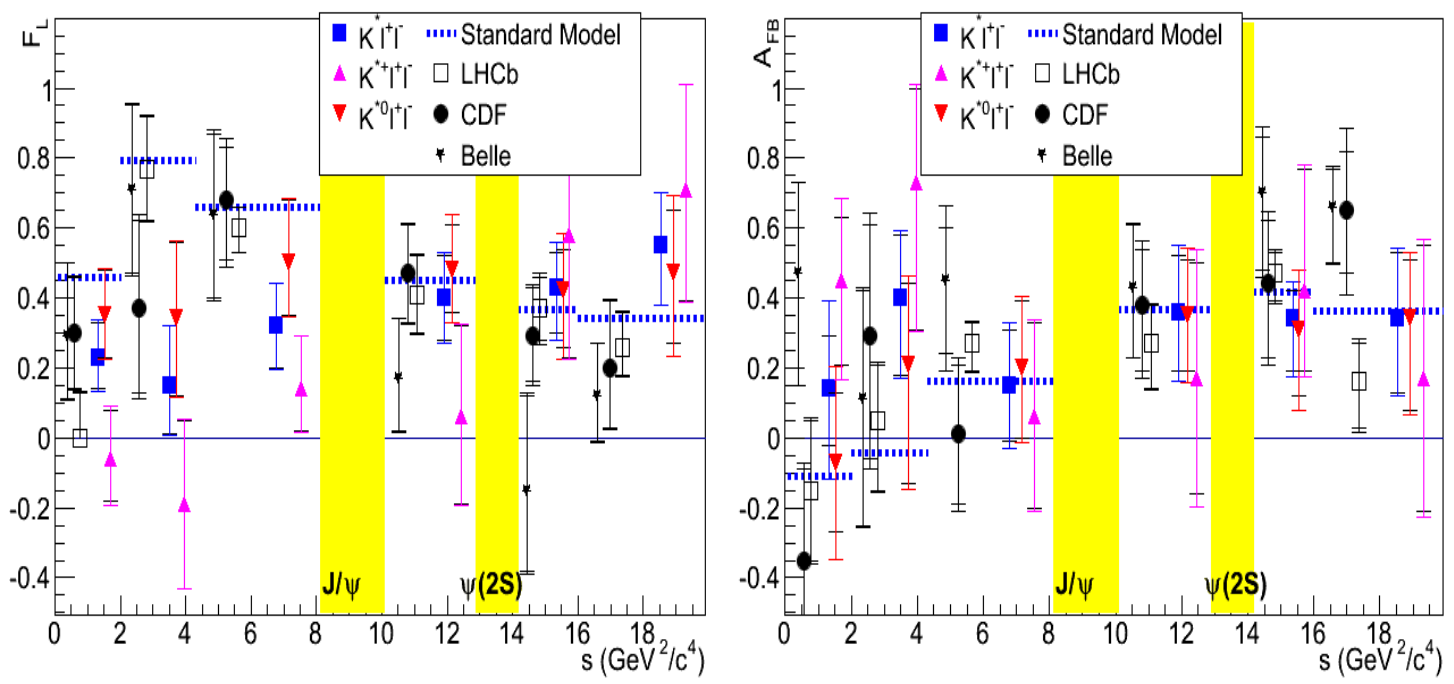

Figure 6: Longitudinal polarization $F_{L}$ (left) and Forward-Backward asymmetry $A_{F B}$ (right) results for $B \rightarrow$ $K^{*} \ell^{+} \ell^{-}$as a function of $s$ for preliminary BABAR measurements (also shown separately for $K^{* 0} \ell^{+} \ell^{-}$and $K^{*+} \ell^{+} \ell^{-}$), Belle [20] (stars), LHCb [22] (open squares), CDF [25] (full circles), and the central value of the SM prediction (dotted line). 


\section{References}

[1] B. Aubert et al. [BABAR Collaboration], Nucl. Instrum. Methods Phys. Res., Sect. A 479, 1 (2002).

[2] A. Abashian et al. [Belle Collaboration], Nucl. Instrum. Methods Phys. Res., Sect. A 479, 1 (2002).

[3] G. Buchalla and A. J. Buras, Nucl. Phys. B 400, 225 (1993).

[4] C. D. Lu and D. X. Zhang, Phys. Lett. B 381, 348 (1996).

[5] K. Nakamura et al. [Particle Data Group], J. Phys. G37, 075021 (2010).

[6] A. Hoecker et al., Proc. Sci., ACAT2007 (2007) 040.

[7] J.P. Lees et al. [BABAR Collaboration], arXiv:1206.3543.

[8] G. Buchalla, A. J. Buras, and M. E. Lautenbacher, Rev. Mod. Phys. 68, 1125 (1996).

[9] W. Altmannshofer, P. Ball, A. Bharucha, A. J. Buras, D. M. Straub and M. Wick, JHEP 0901, 019 (2009).

[10] A. Ali, E. Lunghi, C. Greub, and G. Hiller, Phys. Rev. D 66, 034002 (2002).

[11] F. Kruger, L. M. Sehgal, N. Sinha, and R. Sinha, Phys. Rev. D 61, 114028 (2000) [Erratum-ibid. D 63, 019901 (2001)].

[12] G. Hiller and F. Kruger, Phys. Rev. D 69, 074020 (2004).

[13] Q.-S. Yan, C.-S. Huang, W. Liao, and S.-H. Zhu, Phys. Rev. D 62, 094023 (2000).

[14] T. Feldmann and J. Matias, JHEP 0301, 074 (2003).

[15] B. Aubert et al. [BABAR Collaboration], Phys. Rev. D 70, 112006 (2004).

[16] M. Beneke, T. Feldmann, and D. Seidel, Eur. Phys. Jour. C 41, 173 (2005).

[17] T. Feldmann, 5th Workshop on the CKM Unitary Triangle, Rome (2008).

[18] L. Breiman, Mach. Learn. 24, 123 (1996); I. Narsky, arXiv:physics/0507157 (2005).

[19] J.P. Lees et al. [BABAR Collaboration], arXiv:1204.3933v1.

[20] J.-T. Wei et al. [Belle Collaboration], Phys. Rev. Lett. 103, 171801 (2009).

[21] T. Aaltonen et al. [CDF Collaboration], Phys. Rev. Lett. 107, 201802 (2011);

[22] R. Aaij et al. [LHCb Collaboration], Phys. Rev. Lett. 108, 181806 (2012).

[23] M. Zhong, Y.-L. Wu, and W.-Y. Wang, Int. J. Mod. Phys. A18, 1959 (2003).

[24] P. Ball and R. Zwicky, Phys. Rev. D 71, 014015 (2005); Phys. Rev. D 71, 014029 (2005).

[25] T. Aaltonen et al. [CDF Collaboration], arXiv:1108.0695. 